\title{
QUADRATIC FORMS IN HARMONIC ANALYSIS AND THE BOCHNER-EBERLEIN THEOREM ${ }^{1}$
}

\author{
ROGER A. HORN
}

ABSTRACT. New characterizations are given of the classes of functions on a locally compact Abelian group which are Fourier-Stielties transforms of: bounded measures, nonnegative bounded measures, and integrable functions. These characterizations are all of the same form, viz., that a positive definite Hermitian form dominate a symmetric quadratic form.

1. Introduction. If $G$ is a locally compact Abelian group with dual group $\hat{G}$, we denote by $M(\hat{G})$ the convolution algebra of bounded complex regular measures on $\hat{G}$. We write the group operations in $G$ and $\hat{G}$ additively, denote the identity element of $G$ by 0 , and write the action of a character $\gamma \in \hat{G}$ upon an element $x \in G$ as $(x, y)$. If $\mu \in M(\hat{G})$, we denote the Fourier-Stieltjes transform of $\mu$ by $\hat{\mu}(x)=\int_{\hat{G}}(x, \gamma) d \mu(\gamma)$. We denote Haar measure on $G$ by $d x$ and on $\hat{G}$ by $d \gamma$, and we define $B(G)=\{\hat{\mu} \mid \mu \in M(\hat{G})\}, P(G)=\{\hat{\mu} \mid \mu \in M(\hat{G})$ is a nonnegative measure $\}$, and $A_{p}(G)=\left\{\hat{\mu} \mid d \mu=F d y\right.$ for some $F \in L_{1}(\hat{G}) \cap$ $\left.L_{p}(\hat{G})\right\}, 1 \leq p \leq \infty$. The Fourier transform of a function $f \in L_{1}(G)$ is denoted by $f(y)=\int_{G}(-x, y) f(x) d x$. We refer to [7] for the basic facts about abstract harmonic analy sis.

A complex valued function on $G$ is said to be a positive definite function if

$$
\sum_{i, j=1}^{n} \phi\left(x_{i}-x_{j}\right) c_{i} \bar{c}_{j} \geq 0
$$

for all $x_{1}, \ldots, x_{n} \in G$, all $c_{1}, \ldots, c_{n} \in \mathrm{C}$, and all $n=1,2, \ldots$ Bochner's classical characterization of $P(G)[2]$ is that it is precisely the set of continuous positive definite functions on $G$. Bochner's characterization of $B(G)$ $[3]$ is of a very different nature, however. It does not use the elegant inequalities (1.1) and it does not identify the elements of $P(G) \cap B(G)$. Although (1.1) implies that $\phi$ is continuous on $G$ if it is continuous at 0 , the usual characterization of $B(G)$ requires the a priori assumption of continuity everywhere on $G$.

Presented to the Society, August 8, 1974; received by the editors August 12, 1974. AMS (MOS) subject classifications (1970). Primary 43A25, 43A35, 43A20, 42A72, 42A88, 60E 05 .

Key words and phrases. Fourier-Stieltjes transforms, positive definite functions, Bochner's theorem, Eberlein's theorem.

1 Research supported in part by NSF Grant GP 34638. 
It is the purpose of this note to present simple and similar characterizations of $A_{p}(G), B(G)$, and $P(G)$ which are in the spirit of the inequalities (1.1). In the following section we list some basic facts about quadratic forms and state our characterization theorem; the proof and some remarks follow in the last three sections.

2. Hermitian-symmetric inequalities. If $A=\left(a_{i j}\right)$ and $B=\left(b_{i j}\right)$ are $n \times n$ complex matrices, we say that $A \succ_{S} B$ if $B$ is symmetric and

$$
\sum_{i, j=1}^{n} a_{i j} c_{i} \bar{c}_{j} \geq\left|\sum_{i, j=1}^{n} b_{i j} c_{i} c_{j}\right|
$$

for all $c_{1}, \ldots, c_{n} \in \mathbf{C}$. If $C=\left(c_{i j}\right)$ and $D=\left(d_{i j}\right)$ are complex matrices with the same dimensions, we define the Schur product $C \circ D \equiv\left(c_{i j} d_{i j}\right)$. We write $\operatorname{det} A$ for the determinant if $A$ is a square matrix.

If $B \neq 0$, the relation $A>_{S} B$ imposes conditions on $A$ which are stronger than ordinary positive definiteness. This relation is pregnant with interesting consequences, but we quote here only those results from [5] which are required for the purposes of this note.

Lemma 2.1. Let $A, B, C, D$ be $n \times n$ complex matrices.

(a) If $A=\left(a_{i j}\right)$ and $B=\left(b_{i j}\right)$, if $B$ is symmetric, and if $A>_{S} B$, then

(i) $A$ is a Hermitian positive semidefinite matrix,

(ii) $a_{i i} a_{j j} \geq \max \left(\left|a_{i j}\right|^{2},\left|b_{i j}\right|^{2}\right)$ for all $i, j=1, \ldots, n$,

(iii) $\operatorname{det} A \geq|\operatorname{det} B|$, and

$$
\left|\sum_{i, j=1}^{n} b_{i j} x_{i} y_{j}\right|^{2} \leq\left(\sum_{i, j=1}^{n} a_{i j} x_{i} \bar{x}_{j}\right)\left(\sum_{i, j=1}^{n} a_{i j} y_{i} \bar{y}_{j}\right) \text { for all } x, y \in \mathbf{C}^{n} \text {. }
$$

(b) If $A>_{S} B$ and $C \succ_{S} D$, if $B$ and $D$ are symmetric, and if $\alpha_{i}, \beta_{i}$ $\epsilon \mathbf{C}$ are such that $a_{i} \geq\left|\beta_{i}\right|$ for $i=1,2$ then

(i) $A \circ C \succ_{S} B \circ D$, and

(ii) $a_{1} A+a_{2} C>_{s} \beta_{1} B+\beta_{2} D$.

We are here interested in a very special type of Hermitian-symmetric domination: If $f$ and $\phi$ are complex valued functions on an Abelian group $G$, we say that $\phi(x-y)>_{S} f(x+y)$ on $G$ if

$$
\sum_{i, j=1}^{n} \phi\left(x_{i}-x_{j}\right) c_{i} \bar{c}_{j} \geq\left|\sum_{i, j=1}^{n} f\left(x_{i}+x_{j}\right) c_{i} c_{j}\right|
$$

for all $x_{1}, \ldots, x_{n} \in G$, all $c_{1}, \ldots, c_{n} \in \mathrm{C}$, and all $n=1,2, \ldots$ A function $\phi$ is positive definite $(1.1)$ if $\phi(x-y)>_{S} 0$ on $G$. As a direct corollary of Lemma 2.1 we have

Lemma 2.2. Let $G$ be an Abelian group, and let $\phi$ and $f$ be complex valued functions on $G$. 
(a) If $\phi(x-y)>_{s} f(x+y)$ on $G$ then

(i) $\phi$ is a positive definite function on $G$,

(ii) $\phi(0) \geq \max (|\phi(x)|,|f(x)|)$ for all $x \in G$, and

(iii) $\left|\sum_{i=1}^{n} f\left(x_{i}\right) c_{i}\right|^{2} \leq \phi(0) \sum_{i, j=1}^{n} \phi\left(x_{i}-x_{j}\right) c_{i} \bar{c}_{j}$ for all $x_{1}, \ldots, x_{n} \epsilon$ $G$, all $c_{1}, \ldots, c_{n} \in \mathrm{C}$, and all $n=1,2, \ldots$.

(b) If $\phi_{i}(x-y) \succ_{S} f_{i}(x+y)$ on $G$ and if $\alpha_{i}, \beta_{i} \in \mathbf{C}$ are such that $\alpha_{i}$ $\geq\left|\beta_{i}\right|$ for $i=1,2$, then

(i) $\left(\phi_{1} \phi_{2}\right)(x-y)>_{s}\left(f_{1} f_{2}\right)(x+y)$ on $G$, and

(ii) $\left(\alpha_{1} \phi_{1}+\alpha_{2} \phi_{2}\right)(x-y)>_{S}\left(\beta_{1} f_{1}+\beta_{2} f_{2}\right)(x+y)$ on $G$.

(c) If $G$ is a topological Abelian group, if $\phi(x-y) \succ_{S} f(x+y)$ on $G$, and if $\phi$ and $f$ are both continuous at 0 , then $\phi$ and $f$ are both uniformly continuous on $G$.

(d) If $G$ is a locally compact Abelian group with Haar measure $d x$, if $\phi(x-y) \succ_{S} f(x+y)$ on $G$, and if $\phi$ and $f$ are both continuous at 0 , then

$$
\begin{aligned}
\left|\iint_{G \times G} f(x+y) h(x) h(y) d x d y\right| & \leq \iint_{G \times G} \phi(x-y) h(x) \bar{h}(y) d x d y \text {, and } \\
\left|\int_{G} f(x) h(x) d x\right|^{2} & \leq \phi(0) \iint_{G \times G} \phi(x-y) h(x) \bar{h}(y)
\end{aligned}
$$

for all $h \in L_{1}(G)$.

Proof. If $\phi(x-y)>_{S} f(x+y)$ on $G$, then it is immediate from Lemma 2.1(a-i, ii) that $\phi$ is a positive definite function and that both $\phi$ and $f$ are bounded by $\phi(0)$. Using Lemma $2.1(\mathrm{a}-\mathrm{iv})$ we see that

$$
\left|\sum_{i, j=0}^{n} f\left(x_{i}+x_{j}\right) c_{i} d_{j}\right|^{2} \leq\left(\sum_{i, j=0}^{n} \phi\left(x_{i}-x_{j}\right) c_{i} \bar{c}_{j}\right)\left(\sum_{i, j=0}^{n} \phi\left(x_{i}-x_{j}\right) d_{i} \bar{d}_{j}\right)
$$

for all $x_{0}, x_{1}, \ldots, x_{n} \in G$, all $c_{0}, d_{0}, \ldots, c_{n}, d_{n} \in \mathrm{C}$, and all $n=0,1,2$, .... The inequality in (a-iii) results if we set $x_{0}=0, d_{0}=1, c_{0}=0$, and $d_{1}=d_{2}=\cdots=d_{n}=0$ in (2.3). The assertions in (b) follow directly from Lemma 2.1(b). If we choose $n=3, x_{1}=0, x_{2}=x$, and $x_{3}=y$ in (2.2) and apply the determinant inequality in Lemma $2.1(\mathrm{a}-\mathrm{iii})$ we obtain the inequality

$$
\begin{aligned}
& \phi(0)\left[\phi^{2}(0)-|\phi(y)|^{2}\right]-2 \operatorname{Re} \phi(-x) \phi(x-y)[\phi(0)-\phi(y)]-\phi(0)|\phi(x)-\phi(x-y)|^{2} \\
& \geq \mid-f(0)[f(x)-f(x+y)]^{2}+[f(2 y)-f(0)]\left[f(0) f(2 x)-f^{2}(x)\right] \\
&+[f(y)-f(0)][2 f(x) f(x+y)-f(2 x)\{f(y)+f(0)\}] \mid
\end{aligned}
$$

which becomes

$$
o(1) \geq \phi(0)|\phi(x)-\phi(x-y)|^{2}+|f(0)||f(x)-f(x+y)|^{2} \text { as } y \rightarrow 0
$$

if we invoke boundedness of $f$ and $\phi$ and continuity at 0 ; the $o(1)$ terms are all uniform in $x$. If $\phi(0)=0$ then $\phi(x)=0=f(x)$ for all $x \in G$ by (a-ii). If $f(0)=0$, the above argument can be applied with $\phi$ and $f$ replaced with 
$\phi+1$ and $f+1$, respectively. Finally, (d) follows from (2.2) and (a-iii) with a limiting argument.

The above ideas permit us to state and prove our main result:

Theorem 2.3. Let $G$ be a locally compact Abelian group and let $f$ be a complex valued function on $G$. Then

(a) $f \in P(G)$ if and only if $f$ is continuous at 0 and $f(x-y)>_{S} f(x+y)$ on $G$,

(b) $f \in B(G)$ if and only if $f$ is continuous at 0 and $\phi(x-y)>_{S} f(x+y)$ on $G$ for some $\phi$ which is continuous at zero, and

(c) $f \in A_{p}(G)$ if and only if $f$ is continuous at 0 and $\phi(x-y)>_{S} f(x+y)$ on $G$ for some $\phi \in A_{p}(G), 1 \leq p \leq \infty$.

If we denote by $P^{\prime}(G), B^{\prime}(G)$, and $A_{p}^{\prime}(G)$ the classes of functions which satisfy the conditions stated in (a), (b), and (c) above, respectively, then it follows from Lemma 2.2 that:

(i) Each function in $B^{\prime}(G)$ is bounded and continuous;

(ii) $B^{\prime}(G)$ and $A_{p}^{\prime}(G)$ are linear spaces and $P^{\prime}(G)$ is a convex cone;

(iii) $A_{p}^{\prime}(G), B^{\prime}(G)$, and $P^{\prime}(G)$ are all closed under ordinary multiplication of functions; and

(iv) $P^{\prime}(G)$ is closed under uniform limits.

3. Bochner's theorem and $P(G)$. The fundamental characterization of $P(G)$ is due to Toeplitz [9] when $G=Z$, to Bochner [2] when $G=\mathbf{R}$, and to Weil $[10]$ in the general case:

Theorem 3.1. Let $G$ be a locally compact Abelian group and let $\phi$ be a complex valued function on $G$. Then $\phi \in P(G)$ if and only if $\phi$ is a positive definite function on $G$ which is continuous at 0 .

If $\phi=\hat{\mu}$ for some nonnegative measure $\mu \in M(\hat{G})$, then

$$
\begin{aligned}
\sum_{i, j=1}^{n} \phi\left(x_{i}-x_{j}\right) c_{i} \bar{c}_{j} & =\sum_{i, j=1}^{n} \int_{\hat{G}}\left(x_{i}-x_{j}, \gamma\right) c_{i} \bar{c}_{j} d \mu(\gamma)=\int_{\hat{G}}\left|\sum_{i=1}^{n} c_{i}\left(x_{i}, \gamma\right)\right|^{2} d \mu(\gamma) \\
& \geq\left|\int_{\hat{G}}\left(\sum_{i=1}^{n} c_{i}\left(x_{i}, \gamma\right)\right)^{2} d \mu(\gamma)\right|=\left|\int_{\hat{G}} \sum_{i, j=1}^{n}\left(x_{i}+x_{j}, \gamma\right) c_{i} c_{j} d \mu(\gamma)\right| \\
& =\left|\sum_{i, j=1}^{n} \phi\left(x_{i}+x_{j}\right) c_{i} c_{j}\right|,
\end{aligned}
$$

and hence $\phi(x-y) \succ_{s} \phi(x+y)$ on $G$.

Conversely, if $\phi(x-y)>_{S} \phi(x+y)$ on $G$ then we know from Lemma 2.2(a-i) that $\phi$ is a positive definite function. This brief argument shows that: 
If $\phi \in P(G)$ then $\phi(x-y)>_{s} \phi(x+y)$ on $G$. If $\phi$ is continuous at zero and $\phi(x-y)>_{S} 0$ on $G$, then $\phi \in P(G)$.

4. The Bochner-Eberlein theorem and $B(G)$. The fundamental characterization of $B(G)$ is due to Bochner [3] when $G=\mathbf{R}$ and to Eberlein [4] in the general case:

Theorem 4.1. Let $G$ be a locally compact Abelian group and let $f$ be a complex valued function on $G$. Then $f \in B(G)$ if and only if $f$ is continuous on $G$ and

$$
\left|\sum_{i=1}^{n} c_{i} f\left(x_{i}\right)\right| \leq K \sup _{\gamma \in \hat{G}}\left|\sum_{i=1}^{n} c_{i}\left(x_{i}, \gamma\right)\right|
$$

for all $x_{1}, \ldots, x_{n} \in G$, all $c_{1}, \ldots, c_{n} \in \mathbf{C}$, and all $n=1,2, \ldots$.

If we denote the total variation measure of a measure $\mu \in M(\hat{G})$ by $|\mu|$ and its total variation norm by $\|\mu\|=\int_{\hat{G}} d|\mu|$, and if $f$ satisfies (4.1), then it is known that $f=\hat{\mu}$ for some $\mu \in M(\hat{G})$ and $\|\mu\|=\inf \{K \mid$ equation (4.1) is satisfied $\}$.

If $f=\hat{\mu}$ for some $\mu \in M(\hat{G})$, then using the Jordan decomposition of the real and imaginary parts of $\mu$ we can write $f=a_{1} \mu_{1}+\cdots+a_{4} \mu_{4}$ for some $a_{1}, \ldots, a_{4} \in \mathbf{C}$ and some nonnegative measures $\mu_{1}, \ldots, \mu_{4} \in M(\hat{G})$. Thus, for all $x_{1}, \ldots, x_{n} \in G$, all $c_{1}, \ldots, c_{n} \in \mathrm{C}$, and all $n=1,2, \ldots$ we have

$$
\begin{aligned}
\left|\sum_{i, j=1}^{n} f\left(x_{i}+x_{j}\right) c_{i} c_{j}\right| & =\left|\sum_{k=1}^{4} a_{k} \sum_{i, j=1}^{n} \hat{\mu}_{k}\left(x_{i}+x_{j}\right) c_{i} c_{j}\right| \\
& \leq \sum_{k=1}^{4}\left|a_{k}\right|\left|\sum_{i, j=1}^{n} \hat{\mu}_{k}\left(x_{i}+x_{j}\right) c_{i} c_{j}\right| \\
& \leq \sum_{k=1}^{4}\left|a_{k}\right| \sum_{i, j=1}^{n} \hat{\mu}_{k}\left(x_{i}-x_{j}\right) c_{i} \bar{c}_{j}=\sum_{i, j=1}^{n} \phi\left(x_{i}-x_{j}\right) c_{i} \bar{c}_{j}
\end{aligned}
$$

where $\phi \equiv\left|a_{1}\right| \hat{\mu}_{1}+\cdots+\left|a_{4}\right| \hat{\mu}_{4} \in P(G)$ and we have used Theorem 2.3(a).

Conversely, if $f$ and $\phi$ are complex valued functions on $G$ which are continuous at 0 and if $\phi(x-y)>_{S} f(x+y)$ on $G$, then by Lemma 2.2(a, c) and Theorem 3.1 we know that $f$ and $\phi$ are uniformly continuous on $G, \phi \epsilon$ $P(G)$, and

$$
\left|\sum_{i=1}^{n} f\left(x_{i}\right) c_{i}\right|^{2} \leq \phi(0) \sum_{i, j=1}^{n} \phi\left(x_{i}-x_{j}\right) c_{i} \bar{c}_{j}
$$

for all $x_{1}, \ldots, x_{n} \in G$, all $c_{1}, \ldots, c_{n} \in \mathrm{C}$, and all $n=1,2, \ldots$. But since $\phi=\hat{\nu}$ for some nonnegative measure $\nu \in M(\hat{G})$ we also have 


$$
\begin{aligned}
\sum_{i, j=1}^{n} \phi\left(x_{i}-x_{j}\right) c_{i} \bar{c}_{j} & =\sum_{i, j=1}^{n} \int_{\hat{G}}\left(x_{i}-x_{j}, \gamma\right) c_{i} \bar{c}_{j} d \nu(\gamma) \\
& =\int_{\hat{G}}\left|\sum_{i=1}^{n} c_{i}\left(x_{i}, \gamma\right)\right|^{2} d \nu(\gamma) \leq\|\nu\| \sup _{\gamma_{\epsilon} \hat{G}}\left|\sum_{i=1}^{n} c_{i}\left(x_{i}, \gamma\right)\right|^{2} .
\end{aligned}
$$

Since $\phi(0)=\|\nu\|$ we conclude that (4.1) holds with $K=\phi(0)$. The se observations show that:

$$
\begin{aligned}
& \text { If } f \in B(G) \text { then there exists some } \phi \in P(G) \text { such that } \\
& \phi(x-y) \succ_{S} f(x+y) \text { on } G \text {. If } f \text { is continuous at zero } \\
& \text { and if there exists some } \phi \text { continuous at zero such } \\
& \text { that } \phi(x-y) \succ_{S} f(x+y) \text { on } G \text {, then } f \in B(G) \text {. }
\end{aligned}
$$

Now suppose that $\phi(x-y)>_{S} f(x+y)$ on $G$ and that both $\phi$ and $f$ are continuous at zero; we know then that $\phi=\hat{\mu}$ and $f=\hat{\nu}$ for some $\mu, \nu \in M(\hat{G})$. If we write the inequality in Lemma $2.2(\mathrm{~d}-\mathrm{i})$ in terms of the Fourier transforms, we find that

$$
\left|\int_{\hat{G}}(\hat{h}(\gamma))^{2} d \nu(\gamma)\right| \leq \int_{\hat{G}}|\hat{h}(\gamma)|^{2} d \mu(\gamma)
$$

for all $h \in L_{1}(G)$. Let $E$ be a compact subset of $\hat{G}$ and let $V$ be any open set containing $E$. One knows [7, pp. 48-49] that there exists some $h \in L_{1}(G)$ such that $0 \leq \hat{h}(\gamma) \leq 1$ for all $\gamma \in \hat{G}, \hat{h} \equiv 1$ on $E$ and $\hat{h} \equiv 0$ on the complement of $V$. Using such a function in inequality (4.2), we find that

$$
\left|\nu(E)+\int_{V \sim E}(\hat{h}(\gamma))^{2} d \nu(\gamma)\right| \leq \mu(E)+\int_{V \sim E}(\hat{h}(\gamma))^{2} d \mu(\gamma)
$$

where we denote the relative complement of $E$ in $V$ by $V \sim E$.

Thus for each compact set $E \subset \hat{G}$ and every open set $V \supset E$ we have $|\nu(E)| \leq \mu(E)+(\mu+|\nu|)(V \sim E)$. But since $\mu$ and $\nu$ are regular measures, the open set $V$ can be chosen so as to make the last term arbitrarily small. We conclude that $|\nu(E)| \leq \mu(E)$ for every compact set $E$, and hence that $|\nu(B)| \leq \mu(B)$ and $|\nu|(B) \leq \mu(B)$ for every Borel set $B$. These measure inequalities imply that

$$
\left|\int_{\hat{G}} g(\gamma) d \nu(\gamma)\right| \leq \int_{\hat{G}}|g(\gamma)| d|\nu|(\gamma) \leq \int_{\hat{G}}|g(\gamma)| d \mu(\gamma)
$$

for all $g \in L_{1}(\hat{G},|\nu|)$, and hence that

$$
\left|\int_{G} f(x) h(x) d x\right|=\left|\int_{\hat{G}} \hat{H}(\gamma) d \nu(\gamma)\right| \leq \int_{\hat{G}}|\hat{H}(\gamma)| d \mu(\gamma)=\int_{G} \phi(x) h^{\dagger}(x) d x
$$

for all $h \in L_{1}(G) \cap B(G)$. We write $H(x) \equiv h(-x)$ and denote the inverse 
Fourier transform of the function $|\hat{H}|$ by $h^{\dagger}$; if $h \in L_{1}(G) \cap P(G)$ then $h^{\dagger}=h$.

The inequality $|\nu|(B) \leq \mu(B)$ implies that the measure $\nu$ is absolutely continuous with respect to $\mu$; denote the Radon-Nikodym derivative by $d \nu / d \mu$ $\equiv F$. Since $|\nu(B)|=\left|\int_{B} F(\gamma) d \mu(\gamma)\right| \leq \mu(B)=\int_{B} d \mu$ for every Borel set $B$, we must have $\mu\{\gamma \in \hat{G}|| F(\gamma) \mid>1\}=0$. Thus, we may assume that $F \in L_{\alpha^{\prime}}(\hat{G})$ with $\|F\|_{\infty} \leq 1$. Conversely, if $d \nu=F d \mu$ for some bounded measurable function $F$ with $|F| \leq 1$ on the support of $\mu$, then it follows easily that $\hat{\mu}(x-y)>_{S} \hat{\nu}(x+y)$ on $G$. We summarize these observations in

Theorem 4.1. Let $\phi$ and $f$ be complex valued functions on a locally compact Abelian group $G$, and let both $\phi$ and $f$ be continuous at zero. Then:

(a) $\phi(x-y)>_{s} f(x+y)$ on $G$ if and only if there exists a nonnegative measure $\mu \in M(\hat{G})$ and a function $F \in L_{\infty}(\hat{G})$ such that $\mu\{|F|>1\}=0, \phi=\hat{\mu}$, and $f=\hat{\nu}$ where $d \nu=F d \mu$.

(b) $\phi(x-y)>_{S} f(x+y)$ on $G$ if and only if $f=\hat{\nu}$ for some $\nu \in M(\hat{G})$ and $\phi=\hat{\mu}$ where $\mu=|\nu|+\lambda$ for some nonnegative measure $\lambda \epsilon M(\hat{G})$.

(c) If $f=\hat{\nu}$ for some $\nu \in M(\hat{G})$, then $\|\nu\|=\inf \left\{\phi(0) \mid \phi(x-y)>_{S} f(x+y)\right.$ on $G$ and $\phi$ is continuous at zero\}.

(d) If $\phi(x-y)>_{s} f(x+y)$ on $G$, then $\phi=\hat{\mu}$ and $f=\hat{\nu}$ for some $\mu, \nu$ $\in M(\hat{G})$ with the support of $\nu$ contained in the support of $\mu_{0}$

(e) If $\phi(x-y)>_{S} f(x+y)$ on $G$ then $\left|\int_{G} f(x) h(x) d x\right| \leq \int_{G} \phi(x) h^{\dagger}(x) d x$ for all $h \in L_{1}(G) \cap B(G)$.

5. The Berry-Ryan theorems and $A_{p}(G)$. The fundanental characterization of $A_{p}(G)$ is due to Berry [1] when $G=\mathbf{R}$ and $p=1$, and to Ryan [8] in the general case. Using Theorem 4.1, however, we can easily characterize $A_{p}(G), 1 \leq p \leq \infty$, using $A_{p}(G) \cap P(G)$. If $\phi(x-y)>_{S} f(x+y)$ on $G$ and if both $\phi$ and $f$ are continuous at zero, then we know $\phi=\hat{\mu}$ and $f=\hat{\nu}$ with $\mu, \nu \in M(\hat{G}), \mu$ nonnegative, and $d \nu=F d \mu$ for some $F \in L_{\infty}(\hat{G})$. If $\phi \in A_{p}(G)$ then $\mu$ must be absolutely continuous with respect to Haar measure and $d \mu=$ $\Phi d \gamma$ for some $\Phi \in L_{1}(\hat{G}) \cap L_{p}(\hat{G})$. But then $d \nu=F d \mu=F \Phi d \gamma$ and $F \Phi \epsilon$ $L_{1}(\hat{G}) \cap L_{p}(\hat{G})$ so $f \in A_{p}(G)$.

Conversely, if $f \in A_{p}(G)$ then $f=\hat{\nu}$ where $d \nu=\Phi d y$ and $\Phi \in L_{1}(\hat{G}) \cap$ $L_{p}(\hat{G})$. But if we set $\phi \equiv \hat{\mu}$ where $d \mu \equiv d|\nu|=|\Phi| d \gamma$, then $\phi \in A_{p}(G)$ and $\phi(x-y)>_{s} f(x+y)$ by Theorem 4.1(b). These observations show that:

If $f \in A_{p}(G)$ then there exists some $\phi \in A_{p}(G) \cap P(G)$ such that $\phi(x-y) \succ_{S} f(x+y)$ on $G$. If $f$ is continuous at zero and if there exists some $\phi \in A_{p}(G)$ such that $\phi(x-y) \succ_{S} f(x+y)$ on $G$, then $f \in A_{p}(G)$.

It should be noted that it may often be easier to tell whether a given function $\phi \in P(G)$ is also in $A_{p}(G)$ than to tell whether a function $f \in B(G)$ is 
also in $A_{p}(G)$. For example, when $G=\mathbf{R}$ and $p=1$ Pólya's criterion [6] is a simple sufficient condition: $\phi$ is real valued, even, continuous, convex on $(0, \infty)$, and $\phi(x) \rightarrow 0$ as $x \rightarrow \infty$.

\section{REFERENCES}

1. A. C. Berry, Necessary and sufficient conditions in the theory of Fourier transforms, Ann. of Math. 32 (1931), 830-838.

2. S. Bochner, Vorlesungen über Fouriersche Integrale, Akad, Verlagsgesellschaft, Leipzig, 1932; English transl., Ann. of Math. Studies, no. 42, Princeton Univ. Press, Princeton, N. J., 1959. MR 21 \#5851.

3. S. Bochner, $A$ theorem on Fourier-Stieltjes integrals, Bull. Amer. Math. Soc. 40 (1934), 271-276.

4. W. F. Eberlein, Characterizations of Fourier-Stieltjes transforms, Duke Math. J. 22 (1955), 465-468. MR 17, 281.

5. R. Hom, On inequalities between Hermitian and symmetric forms, Linear Algebra and Appl. (to appear).

6. G. Pólya, Remarks on characteristic functions, Proc. Berkeley Sympos. on Math. Statist. and Probability, Univ. of Calif. Press, Berkeley, Calif., 1949, pp. 115-123. MR 10, 463.

7. W. Rudin, Fourier analysis on groups, Interscience, New York, 1962. MR $27 \# 2808$.

8. R. Ryan, Fourier transforms of certain classes of integrable functions, Trans. Amer. Math. Soc. 105 (1962), 102-111. MR 32 \#4475.

9. O. Toeplitz, Über die Fourier'sche Entwickelung positiver Funktionen, Rend. Circ. Mat. Palmermo 32 (1911), 191-192.

10. A. Weil, L'intégration dans les groupes topologiques et ses applications, Actualités Sci. Indust., no 869, 1145, Hermann, Paris, 1941, 1951. MR 3, 198.

DEPARTMENT OF MATHEMATICAL SCIENCES, THE JOHNS HOPKINS UNIVERSITY, BALTIMORE, MARYLAND 21218 